\title{
Crystal structure of $\left(1^{\prime} R^{*}, 2 R^{*}, 3 R^{*}, 4 S^{*}, 5 S^{*}, 6 S^{*}\right)-2,3,4,5$-tetrahydro-6- hydroxy-2-(1'-hydroxyethyl)-3-hydroxymethyl-5-methoxy-2,4- heptanofuran, $\mathrm{C}_{11} \mathrm{H}_{16} \mathrm{O}\left[\mathrm{CH}(\mathrm{OH}) \mathrm{CH}_{3}\right](\mathrm{OH})\left(\mathrm{CH}_{2} \mathrm{OH}\right)\left(\mathrm{OCH}_{3}\right)$
}

\author{
K. Peters, E.-M. Peters
}

Max-Planck-Institut für Festkörperforschung. Heisenbergstraße 1, D-70506 Stuttgart, Germany

M. Kasch and W. Tochtermann

Christian-Albrechts-Universität Kiel, Institut für Organische Chemie, Olshausenstraße 40, D-24098 Kiel, Germany

Received August 14, 1997, CSD-No. 409074

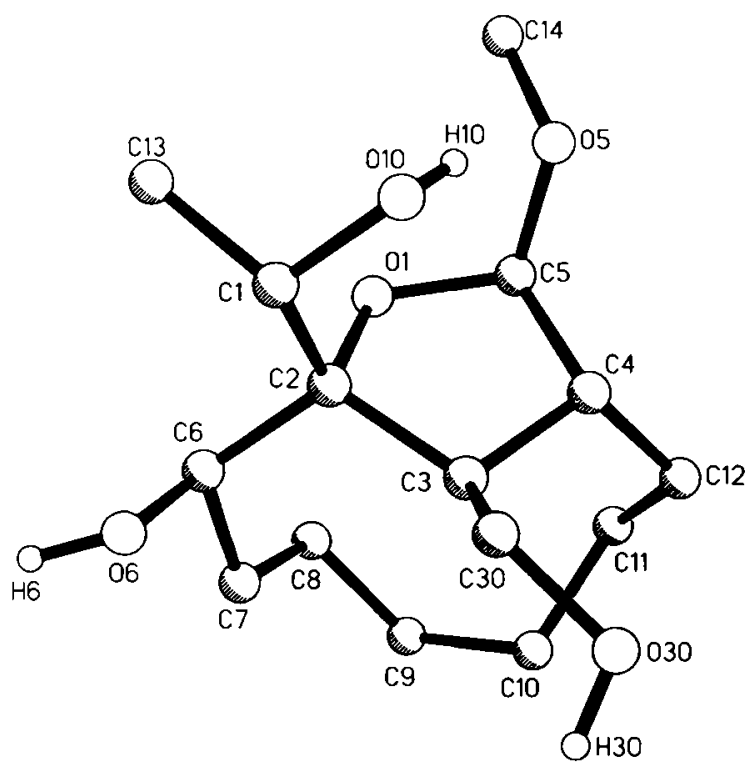

Table 1. Parameters used for the $X$-ray data collection

\begin{tabular}{ll}
\hline Crystal: & colorless plate, size $0.15 \times 0.5 \times 0.75 \mathrm{~mm}$ \\
Wavelength: & Mo $K_{\alpha}$ radiation $(0.71073 \AA)$ \\
$\mu:$ & $0.90 \mathrm{~cm}^{-1}$ \\
Diffractometer: & Siemens P4 \\
Scan mode: & $\omega$ \\
$T_{\text {measurement: }}$ & $293 \mathrm{~K}$ \\
$2 \theta_{\text {max }}$ & $55^{\circ}$ \\
$\mathrm{N}(\text { hkl })_{\text {unique: }}$ & 3561 \\
Criterion for $F_{\mathrm{o}}:$ & $F_{0}>3 \sigma\left(F_{\mathrm{o}}\right)$ \\
$\mathrm{N}(\text { param })_{\text {refined: }}$ & 194 \\
Program: & SHELXTL-plus \\
\hline
\end{tabular}

Source of material: The title compound was prepared as described in ref. 1 .

$\mathrm{C}_{15} \mathrm{H}_{28} \mathrm{O}_{5}$, monoclinic, $P 12{ }_{1} / n 1$ (No. 14), $a=8.021(1) \AA$, $b=21.679(3) \AA, c=9.418(1) \AA, \beta=108.89(1)^{\circ}, V=1549.5 \AA^{3}, Z=4$, $R(F)=0.059, R_{\mathbb{W}}(F)=0.061$.

Table 2. Final atomic coordinates and displacement parameters (in $\AA^{2}$ )

\begin{tabular}{llllll}
\hline Atom & Site & $x$ & $y$ & $z$ & $U_{\text {iso }}$ \\
\hline H(1) & $4 e$ & $-0.0999(3)$ & $0.7789(1)$ & $0.4978(2)$ & 0.08 \\
H(3) & $4 e$ & $0.1426(3)$ & $0.82005(8)$ & $0.8566(2)$ & 0.08 \\
H(4) & $4 e$ & $-0.1685(3)$ & $0.87625(9)$ & $0.8466(2)$ & 0.08 \\
H(5) & $4 e$ & $0.0011(3)$ & $0.97315(9)$ & $0.7768(2)$ & 0.08 \\
H(6A) & $4 e$ & $0.2346(3)$ & $0.85773(9)$ & $0.5424(2)$ & 0.08 \\
H(6) & $4 e$ & $0.288(4)$ & $0.758(1)$ & $0.525(3)$ & $0.072(9)$ \\
H(7A) & $4 e$ & $0.3711(3)$ & $0.8104(1)$ & $0.8277(3)$ & 0.08 \\
H(7B) & $4 e$ & $0.4808(3)$ & $0.8220(1)$ & $0.7194(3)$ & 0.08 \\
H(8A) & $4 e$ & $0.3244(3)$ & $0.9251(1)$ & $0.7889(3)$ & 0.08 \\
H(8B) & $4 e$ & $0.5122(3)$ & $0.9190(1)$ & $0.7727(3)$ & 0.08 \\
H(9A) & $4 e$ & $0.5606(3)$ & $0.9420(1)$ & $1.0160(3)$ & 0.08 \\
H(9B) & $4 e$ & $0.6050(3)$ & $0.8717(1)$ & $1.0145(3)$ & 0.08 \\
H(10A) & $4 e$ & $0.3379(3)$ & $0.8467(1)$ & $1.0425(3)$ & 0.08 \\
H(10B) & $4 e$ & $0.4497(3)$ & $0.8874(1)$ & $1.1771(3)$ & 0.08 \\
H(10) & $4 e$ & $-0.287(5)$ & $0.873(2)$ & $0.545(4)$ & $0.11(1)$ \\
H(11A) & $4 e$ & $0.2308(4)$ & $0.9587(1)$ & $0.9601(3)$ & 0.08 \\
H(11B) & $4 e$ & $0.2529(4)$ & $0.9574(1)$ & $1.1317(3)$ & 0.08 \\
H(12A) & $4 e$ & $0.0524(3)$ & $0.8705(1)$ & $1.0694(3)$ & 0.08 \\
H(12B) & $4 e$ & $-0.0268(3)$ & $0.9370(1)$ & $1.0318(3)$ & 0.08 \\
H(13A) & $4 e$ & $-0.0006(4)$ & $0.8368(1)$ & $0.3366(3)$ & 0.08 \\
H(13B) & $4 e$ & $-0.1270(4)$ & $0.8908(1)$ & $0.3453(3)$ & 0.08 \\
H(13C) & $4 e$ & $-0.2053(4)$ & $0.8281(1)$ & $0.2701(3)$ & 0.08 \\
H(14A) & $4 e$ & $-0.3672(4)$ & $1.0126(1)$ & $0.4952(4)$ & 0.08 \\
H(14B) & $4 e$ & $-0.1849(4)$ & $0.9951(1)$ & $0.4783(4)$ & 0.08 \\
H(14C) & $4 e$ & $-0.1926(4)$ & $1.0392(1)$ & $0.6079(4)$ & 0.08 \\
H(30A) & $4 e$ & $-0.0209(3)$ & $0.73666(9)$ & $0.7481(2)$ & 0.08 \\
H(30B) & $4 e$ & $-0.1900(3)$ & $0.77308(9)$ & $0.7474(2)$ & 0.08 \\
H(30) & $4 e$ & $0.051(4)$ & $0.724(1)$ & $0.982(3)$ & $0.071(9)$ \\
& & & & & \\
& & & & &
\end{tabular}

Table 3. Final atomic coordinates and displacement parameters (in $\AA^{2}$ )

\begin{tabular}{llllllllllr}
\hline Atom & Site & $x$ & $y$ & $z$ & $U_{11}$ & $U_{22}$ & $U_{33}$ & $U_{12}$ & $U_{13}$ & $U_{23}$ \\
\hline $\mathrm{O}(1)$ & $4 e$ & $0.0330(2)$ & $0.91399(6)$ & $0.6330(1)$ & $0.0370(7)$ & $0.0297(6)$ & $0.0392(8)$ & $-0.0014(5)$ & $0.0177(6)$ & $0.0059(5)$ \\
$\mathrm{C}(1)$ & $4 e$ & $-0.1152(3)$ & $0.8228(1)$ & $0.4992(2)$ & $0.042(1)$ & $0.047(1)$ & $0.035(1)$ & $-0.0066(9)$ & $0.0123(9)$ & $-0.0035(9)$ \\
$\mathrm{C}(2)$ & $4 e$ & $0.0387(2)$ & $0.84660(8)$ & $0.6343(2)$ & $0.035(1)$ & $0.0296(9)$ & $0.0322(9)$ & $-0.0025(7)$ & $0.0140(8)$ & $0.0010(7)$ \\
$\mathrm{C}(3)$ & $4 e$ & $0.0268(3)$ & $0.82919(8)$ & $0.7894(2)$ & $0.0343(9)$ & $0.0297(9)$ & $0.0327(9)$ & $-0.0019(7)$ & $0.0164(7)$ & $0.0017(7)$
\end{tabular}


Table 3. (Continued)

\begin{tabular}{|c|c|c|c|c|c|c|c|c|c|c|}
\hline Atom & Site & $x$ & $y$ & $z$ & $U_{11}$ & $U_{22}$ & $U_{33}$ & $U_{12}$ & $U_{13}$ & $U_{23}$ \\
\hline$C(4)$ & $4 e$ & $-0.0531(3)$ & $0.88589(9)$ & $0.8430(2)$ & $0.045(1)$ & $0.033(1)$ & $0.039(1)$ & $0.0014(8)$ & $0.0234(9)$ & $0.0032(8)$ \\
\hline$C(5)$ & $4 e$ & $-0.0563(3)$ & $0.93639(9)$ & $0.7280(2)$ & $0.039(1)$ & $0.032(1)$ & $0.043(1)$ & $0.0022(8)$ & $0.0175(9)$ & $0.0042(8)$ \\
\hline$O(5)$ & $4 e$ & $-0.2338(2)$ & $0.95152(7)$ & $0.6461(2)$ & $0.0400(8)$ & $0.0426(8)$ & $0.064(1)$ & $0.0084(7)$ & $0.0229(7)$ & $0.0187(7)$ \\
\hline$C(6)$ & $4 e$ & $0.2161(3)$ & $0.82909(9)$ & $0.6136(2)$ & $0.041(1)$ & $0.036(1)$ & $0.040(1)$ & $-0.0017(8)$ & $0.0228(9)$ & $-0.0017(8)$ \\
\hline$O(6)$ & $4 e$ & $0.1988(2)$ & $0.76574(7)$ & $0.5684(2)$ & $0.057(1)$ & $0.0423(9)$ & $0.067(1)$ & $-0.0031(7)$ & $0.0368(9)$ & $-0.0137(8)$ \\
\hline$C(7)$ & $4 e$ & $0.3837(3)$ & $0.8363(1)$ & $0.7492(3)$ & $0.036(1)$ & $0.047(1)$ & $0.048(1)$ & $0.0054(9)$ & $0.0200(9)$ & $0.002(1)$ \\
\hline$C(8)$ & $4 e$ & $0.4303(3)$ & $0.9009(1)$ & $0.8157(3)$ & $0.042(1)$ & $0.051(1)$ & $0.053(1)$ & $-0.004(1)$ & $0.014(1)$ & $0.006(1)$ \\
\hline $\mathrm{C}(9)$ & $4 e$ & $0.5122(3)$ & $0.9017(1)$ & $0.9865(3)$ & $0.049(1)$ & $0.055(1)$ & $0.052(1)$ & $-0.001(1)$ & $-0.001(1)$ & $-0.001(1)$ \\
\hline$C(10)$ & $4 e$ & $0.3850(3)$ & $0.8871(1)$ & $1.0717(3)$ & $0.067(2)$ & $0.051(1)$ & $0.039(1)$ & $0.004(1)$ & $0.002(1)$ & $-0.002(1)$ \\
\hline$O(10)$ & $4 e$ & $-0.2809(2)$ & $0.83655(8)$ & $0.5175(2)$ & $0.0359(8)$ & $0.054(1)$ & $0.056(1)$ & $-0.0081(7)$ & $0.0089(7)$ & $-0.0051(8)$ \\
\hline$C(11)$ & $4 e$ & $0.2308(4)$ & $0.9328(1)$ & $1.0428(3)$ & $0.070(2)$ & $0.049(1)$ & $0.048(1)$ & $0.000(1)$ & $0.012(1)$ & $-0.012(1)$ \\
\hline$C(12)$ & $4 e$ & $0.0444(3)$ & $0.9060(1)$ & $1.0065(3)$ & $0.070(2)$ & $0.043(1)$ & $0.041(1)$ & $0.006(1)$ & $0.029(1)$ & $-0.0041(9)$ \\
\hline$C(13)$ & $4 e$ & $-0.1117(4)$ & $0.8468(1)$ & $0.3491(3)$ & $0.067(2)$ & $0.081(2)$ & $0.034(1)$ & $-0.012(1)$ & $0.007(1)$ & $0.001(1)$ \\
\hline$C(14)$ & $4 e$ & $-0.2456(4)$ & $1.0040(1)$ & $0.5486(4)$ & $0.057(2)$ & $0.066(2)$ & $0.093(2)$ & $0.015(1)$ & $0.025(1)$ & $0.045(2)$ \\
\hline$C(30)$ & $4 e$ & $-0.0657(3)$ & $0.76841(9)$ & $0.7970(2)$ & $0.049(1)$ & $0.033(1)$ & $0.049(1)$ & $-0.0040(9)$ & $0.025(1)$ & $0.0043(9)$ \\
\hline$O(30)$ & $4 e$ & $-0.0351(2)$ & $0.75171(7)$ & $0.9505(2)$ & $0.062(1)$ & $0.0452(9)$ & $0.063(1)$ & $0.0134(8)$ & $0.0417(9)$ & $0.0234(8)$ \\
\hline
\end{tabular}

\section{References}

1. Kasch, M.: Racemische und optisch aktive überbrïckte Furanoside als Bausteine zur Synthese modifizierter Nucleoside. Dissertation, Universität Kiel, Germany 1996.

2. Sheldrick, G. M.: Program Package SHELXTL-plus. Release 4.1. Siemens Analytical X-Ray Instruments Inc., Madison (WI 53719), USA 1990. 